\title{
Quantum Field Theory in a Non-Commutative Space: Theoretical Predictions and Numerical Results on the Fuzzy Sphere ${ }^{\star}$
}

\author{
Marco PANERO
}

School of Theoretical Physics, Dublin Institute for Advanced Studies, 10 Burlington Road, Dublin 4, Ireland

E-mail: panero@stp.dias.ie

URL: http://www.stp.dias.ie/ panero/

Received September 29, 2006, in final form November 10, 2006; Published online November 17, 2006

Original article is available at http://www.emis.de/journals/SIGMA/2006/Paper081/

\begin{abstract}
We review some recent progress in quantum field theory in non-commutative space, focusing onto the fuzzy sphere as a non-perturbative regularisation scheme. We first introduce the basic formalism, and discuss the limits corresponding to different commutative or non-commutative spaces. We present some of the theories which have been investigated in this framework, with a particular attention to the scalar model. Then we comment on the results recently obtained from Monte Carlo simulations, and show a preview of new numerical data, which are consistent with the expected transition between two phases characterised by the topology of the support of a matrix eigenvalue distribution.
\end{abstract}

Key words: non-commutative geometry; matrix models; non-perturbative effects; phase transitions

2000 Mathematics Subject Classification: 58B34; 81R60

\section{Introduction}

The study of quantum field theory in non-commutative spaces $[1,2,3,4]$ is based on strong physical motivations, since this formalism is relevant to the description of the quantum Hall effect [5], to certain aspects of string theory $[6,7,8,9,10,11]$, as well as to a possible formulation for a quantum theory of gravity [12].

The most extensively studied non-commutative space is probably the Groenewold-Moyal $\mathbb{R}_{\theta}^{n}$ space, defined as the algebra of functions $\mathcal{A}_{\theta}\left(\mathbb{R}^{n}\right)$ generated by the coordinates $x^{\mu}$ ( $\mu \in$ $\{1,2, \ldots, n\})$ with the commutation relations:

$$
\left[x^{\mu}, x^{\nu}\right]_{*}=i \theta^{\mu \nu},
$$

where, for simplicity, $\theta^{\mu \nu}$ can be taken to be independent of $x$, and $*$ denotes the GroenewoldMoyal star-product. For functions $f, g$ in $\mathcal{A}_{\theta}\left(\mathbb{R}^{n}\right)$, the latter is defined to act as $[13,14,15]$ :

$$
f(x) * g(x)=f(x) e^{\frac{i}{2} \overleftarrow{\partial}_{\mu} \theta^{\mu \nu} \vec{\partial}_{\nu}} g(x)
$$

A number of dramatic implications stem from equation (1), which have been studied in detail in the literature $[16,17,18,19,20,21,15,22,23,24,25,26,28,29,27,30,31,32]$. In particular, some issues which are of strong relevance from the physical point of view include:

\footnotetext{
${ }^{\star}$ This paper is a contribution to the Proceedings of the O'Raifeartaigh Symposium on Non-Perturbative and Symmetry Methods in Field Theory (June 22-24, 2006, Budapest, Hungary). The full collection is available at http://www.emis.de/journals/SIGMA/LOR2006.html
} 
- The "ultra-violet/infra-red (UV/IR) mixing" phenomenon: The effective action describing QFT in a Groenewold-Moyal space diverges when the components of the external momentum along the non-commutative directions vanish. This effect arises from the integration of high-energy modes in non-planar loop diagrams, and is related to the non-locality properties of quantum field theory in a non-commutative space.

- Renormalisability: One of the potentially most dangerous consequences of the UV/IR mixing phenomenon is the fact that it threatens the possibility to renormalise quantum field theories defined in a non-commutative setting. However, the proof of perturbative renormalisability at all orders has been eventually worked out adding an oscillator term to the potential of the scalar model [28, 29, 30, 31, 32].

The theoretical predictions for these models can be compared with non-perturbative results obtained from numerical simulations. In fact, it is possible to define a well-suited lattice formulation of the non-commutative theory as a unitary matrix model [33], which possesses a continuum limit characterised by finite space volume and finite non-commutativity. This formulation allowed to investigate numerically various aspects of these models [34, 35, 36, 37, 38, 39, 40, 41, 42].

A different class of non-commutative spaces is given by fuzzy spaces; the basic idea underlying their construction is to use a finite-dimensional matrix algebra to approximate the infinitedimensional algebra of functions on a manifold. This can be done on even-dimensional co-adjoint orbits of Lie groups, which are symplectic manifolds [43, 44, 45, 46, 47, 48, 49, 50, 51], like the two-sphere $S^{2}$ and the $\mathbb{C} P^{n}$ complex projective spaces.

In the following discussion, we shall concentrate our attention onto the fuzzy two-sphere $S_{F}^{2}$ [43]; it provides the simplest example of a fuzzy space, and depends on two parameters: the dimensionful, real and positive, radius $R$, and a dimensionless, integer-valued cut-off, which is associated with the value of the maximum angular momentum $l_{\max }$. In particular, the ordinary, commutative algebra of functions on the sphere is replaced by a non-commutative counterpart, obtained truncating the expansion of functions in the basis of irreducible representations of the $s u(2)$ algebra to a maximum angular momentum $l_{\max }$. This geometrical construction of $S_{F}^{2}$ offers a natural representation for functions on the sphere in terms of matrices belonging to $\operatorname{Mat}_{N}(\mathbb{C})$, with $N=l_{\text {max }}+1^{1}$.

The fuzzy approach explicitly maintains the symmetries of the original manifold for every value of $N$, and it accommodates various topological features in a natural way - see [50] and references therein for details. It provides a well-defined, non-perturbative regularisation scheme for quantum field theory, and - at least in principle - can be considered as a potential alternative to the lattice discretisation. The representation of fields on the fuzzy sphere in terms of finitedimensional matrices also opens up the possibility to study interacting theories via numerical simulations: this has motivated various recent works [52, 53, 54, 55, 56, 57, 58, 59].

Indeed, the fuzzy models reveal a rich and complex structure, encoding a number of interesting properties, which show up even for the simplest theories - e.g.: for a scalar field with quartic interactions.

The aim of this paper consists in presenting an overview on some of these aspects, comparing theoretical expectations and results of Monte Carlo simulations, and highlighting their physical interpretation in different limits.

The sections below are organised according to the following structure. In Section 2, we first introduce the general formalism for the construction of the fuzzy sphere, and discuss its different geometrical limits; then we define the description of QFT on the fuzzy sphere, and present the theoretical predictions which are expected in different limits. Next, the theoretical predictions are compared with non-perturbative results obtained from numerical simulations in

\footnotetext{
${ }^{1}$ This construction can be easily obtained by taking the tensor product of two vector representations of angular momentum $\frac{l_{\max }}{2}$.
} 
Section 3. Finally, in Section 4 we discuss the relevance of these results for the program to use the the fuzzy approach as a regularisation scheme for quantum field theory in commutative or in non-commutative spaces, and conclude with some brief remarks.

\section{General formalism and theoretical predictions}

The construction of a fuzzy space is based on the quantisation of the algebra of functions defined on the corresponding (commutative) manifold. For the two-sphere, the construction can be summarised as it follows [60]: The infinite-dimensional algebra of polynomials in the $\left\{x_{i}\right\}_{i=1,2,3}$ commutative coordinates on the two-dimensional sphere $x_{i} x_{i}=R^{2}$ embedded in $\mathbb{R}^{3}$ is replaced by the non-commutative algebra generated by $\left\{\hat{x}_{i}\right\}_{i=1,2,3}$ operators, which obey the (rescaled) commutation relations of the $s u(2)$ algebra:

$$
\left[\hat{x}_{i}, \hat{x}_{j}\right]=\frac{2 R}{\sqrt{N^{2}-1}} i \epsilon_{i j k} \hat{x}_{k}, \quad \text { with: } \quad \sum_{i=1}^{3} \hat{x}_{i}^{2}=R^{2} .
$$

On the fuzzy sphere, the counterpart of functions defined on the commutative sphere are matrices belonging to $\operatorname{Mat}_{N}(\mathbb{C})$; a natural mapping can be defined truncating the basis of harmonic functions on $S^{2}$ to a maximum value for the angular momentum $l_{\max }$, and associating the harmonic functions with total and third-component angular momentum quantum numbers $l$ and $m$ to the $\left\{\hat{Y}_{l, m}\right\}$ polarisation tensors satisfying:

$$
\left[L_{i},\left[L_{i}, \hat{Y}_{l, m}\right]\right]=l(l+1) \hat{Y}_{l, m}, \quad\left[L_{3}, \hat{Y}_{l, m}\right]=m \hat{Y}_{l, m},
$$

where the $\left\{L_{i}\right\}_{i=1,2,3}$ are the generators of the $s u(2)$ algebra in the $N \times N$ matrix representation. This mapping choice, however, is not unique - a convenient alternative mapping being the one defined in terms of coherent states.

Integrals of functions on the commutative sphere are replaced by the matrix trace operation: given any $\Phi$ and $\Psi$ elements in $\operatorname{Mat}_{N}(\mathbb{C})$, an inner product on $S_{F}^{2}$ is built as:

$$
\langle\Phi, \Psi\rangle=\frac{4 \pi R^{2}}{N} \operatorname{tr}\left(\Phi^{\dagger} \Psi\right)
$$

The vectors describing derivations on the commutative sphere are represented through the adjoint action of the $\left\{L_{i}\right\}_{i=1,2,3}$ generators:

$$
\left[L_{i}, \Phi\right] \text {. }
$$

It is important to discuss what is the meaning of the "commutative limit" in this context: The matrix algebra underlying the fuzzy space is truly non-commutative for any $N$; however, the right-hand side of equation (2) vanishes in the $N \rightarrow \infty$ limit, keeping $R$ fixed. Therefore, given a smooth function defined on the commutative sphere, whose expansion in the spherical harmonics' basis involves non-negligible coefficients up to a certain angular momentum only, the effect of this discretisation will be vanishing in the large- $N$ limit at fixed $R$.

So far, the interpretation of this limit is only concerned with the geometric properties of the manifold - not with the physical properties of a quantum field theory defined in this setting.

Indeed, the quantum features of a physical field theory spoil the correspondence between the fixed- $R$, large- $N$ limit of the (naive formulation of the) theory on the fuzzy sphere, and the corresponding model on the commutative manifold [61].

This can be seen in a perturbative calculation for the (real) scalar model with quartic interactions; for this model, the Euclidean action on the fuzzy sphere can be written in the following form:

$$
S(\Phi)=\frac{4 \pi}{N} \operatorname{tr}\left(\frac{1}{2}\left[L_{i}, \Phi\right]^{\dagger}\left[L_{i}, \Phi\right]+\frac{\mu^{2}}{2} \Phi^{2}+\frac{g}{4 !} \Phi^{4}\right)
$$


(where $\Phi \in \operatorname{Mat}_{N}(\mathbb{C})$ is taken to be Hermitian). At one-loop order in a perturbative expansion, the non-commutative nature of the model manifests itself in the comparison between the contributions to the one-particle-irreducible two-point function from planar $\left(I_{\mathrm{P}}\right)$ and non-planar $\left(I_{\mathrm{NP}}\right)$ diagrams - they differ by a quantity which is finite, non-vanishing, and which has a smooth dependence on $\frac{1}{N}$ :

$$
I_{\mathrm{NP}}=I_{\mathrm{P}}-\frac{2}{N^{2}-1} \sum_{j=0}^{N-1} \frac{j(j+1)(2 j+1)}{j(j+1)+\mu^{2}} .
$$

When the $N \rightarrow \infty$, fixed- $R$, limit is taken, this difference between $I_{\mathrm{NP}}$ and $I_{\mathrm{P}}$ has an impact on the one-loop effective action, as it induces a weakly non-local, $l$-dependent, deformation of the dispersion relation on the fuzzy sphere. This effect has no analogue in the commutative setting, and is therefore called the "non-commutative anomaly".

In order to recover the correct model in the commutative limit, one should redefine the interaction term in in equation (3) via a normal-ordering prescription [62], cancelling the undesired momentum-dependent quadratic terms in the effective action; alternatively, one could include rotationally symmetric higher derivative terms in the matrix action [63].

On the contrary, studying the model defined by the (naive) matrix action in equation (3), one expects to observe deviations with respect to the quantum theory on the commutative sphere. Although the derivation of the non-commutative anomaly in [61] was worked out for the $\mu^{2}>0$ case, one may expect that a similar phenomenon occurs when $\mu^{2}<0$, too.

For QFT on the commutative sphere, the latter regime is particularly interesting, as it corresponds to a situation in which, classically, the $\Phi \rightarrow-\Phi$ symmetry of the model is spontaneously broken. The situation from the quantum point of view is different, however, because - due to the finite size of the system - tunneling events characterised by a finite Euclidean action connect the classical vacua, and the true quantum ground state is actually unique for any finite value of the parameters. Therefore, strictly speaking, one does not expect phase transitions on the commutative sphere with fixed, finite radius; this, however, might no longer be the case for the $N \rightarrow \infty$ limit of the fuzzy model, if "anomalous" effects take place.

For the quantum theory on the commutative sphere, it is also interesting to note that the tunneling phenomenon between the (uniform) classical vacua can be mediated by typical quantum events which break the $S O(3)$ symmetry - for instance, through a $p$-wave field configuration (with axial symmetry only). A signature of these events would show up through non-vanishing expectation values for the modes associated with non-vanishing momenta. In the commutative setting, this phenomenon is possible because of the finite size of the system; on the contrary, one would not expect a non-uniform breaking symmetry pattern in the infinite commutative plane.

Another important implication of equation (4) is that the contribution of non-perturbative diagrams to the two-point function is not divergent for vanishing values of the momentum. This has to be compared and contrasted with the non-commutative plane setting, where the UV/IR mixing phenomenon occurs. Yet, the divergence which is encountered in the the GroenewoldMoyal space is exactly recovered, once a different scaling limit of the fuzzy sphere is considered: this is discussed below.

A local approximation to the non-commutative $\mathbb{R}_{\theta}^{2}$ plane can be obtained, in the following double scaling limit of the fuzzy sphere:

$$
N \rightarrow \infty, \quad R \rightarrow \infty, \quad \text { with: } \quad \theta=\frac{2 R^{2}}{N} \text { fixed }
$$

This can be seen considering a stereographic projection map from the sphere to the $\left(\hat{y}_{1}, \hat{y}_{2}\right)$ plane:

$$
\hat{y}_{+}=\hat{y}_{1}+i \hat{y}_{2}=2 R \hat{x}_{+}\left(R-\hat{x}_{3}\right)^{-1}, \quad \hat{y}_{-}=\hat{y}_{1}-i \hat{y}_{2}=2 R\left(R-\hat{x}_{3}\right)^{-1} \hat{x}_{-} .
$$


In the limit (5), $\hat{y}_{1}$ and $\hat{y}_{2}$ satisfy:

$$
\left[\hat{y}_{1}, \hat{y}_{2}\right]=-i \theta
$$

and the non-commutative anomaly induces a logarithmic infrared divergence proportional to $\log (p \theta)$ in the non-planar contribution to the 1PI two-point function. This divergence is exactly the same which is found in the perturbative calculation for the quantum theory defined in the Groenewold-Moyal plane [22], therefore in this case the (double scaling) large- $N$ limit of the fuzzy sphere reproduces the expected result, and the fuzzy space can be used as a regularisation for non-commutative spaces. In principle, the construction can be generalised to higher dimensional cases, using fuzzy $\mathbb{C} P^{n}$ spaces, or direct products of fuzzy spheres.

The regularisation of Euclidean scalar field theory in even-dimensional, non-commutative $\mathbb{R}_{\theta}^{n}$ spaces by means of fuzzy manifolds was used in [64]. There, the properties of the model were investigated non-perturbatively in a matrix representation for the field, and a critical line corresponding to a phase transition in the associated eigenvalue distribution was derived in the large-matrix limit.

This transition is a change in the topology of the support of the eigenvalue distribution: for values of $\mu^{2}$ above a critical value ("single-cut phase"), the eigenvalue distribution $\rho(\phi)$ has a connected support of finite width, centred in zero, and would reduce to Wigner's semi-circle law in the non-interacting case:

$$
\rho(\phi)=\frac{1}{2 \pi}\left[g_{\mathrm{eff}}\left(\phi^{2}+\frac{1}{2}\right)+\mu_{\mathrm{eff}}^{2}\right] \sqrt{1-\phi^{2}}, \quad \text { with: } g_{\mathrm{eff}}=\frac{4}{3}\left(4-\mu_{\mathrm{eff}}^{2}\right),
$$

where $g_{\text {eff }}$ and $\mu_{\text {eff }}^{2}$ depend on (the renormalised values of) $g$ and $\mu^{2}$.

By contrast, for values of $\mu^{2}$ which are negative and large in modulus, there exist two symmetric, disconnected regions of the real axis with non-vanishing probability for the eigenvalues ("two-cut phase"). In particular, the probability density for the zero eigenvalue vanishes when $\mu^{2}$ is decreased down to the critical point, and for lower $\mu^{2}$ values a gap opens up in the distribution. This phenomenon (which would not take place for a model in ordinary commutative space) is related to the UV/IR mixing effect; it is predicted on the basis of a matrix model description, which relies on the dominance of planar diagrams over the non-planar ones, and can be interpreted as a manifestation of the "striped phase" [24, 25].

Figs. 1 to 4 show $\rho(\phi)$ for different cases. Fig. 1 corresponds to the pure Wigner's semi-circle law; next, Fig. 2 displays the eigenvalue density profile in the "one-cut" regime. When $\mu_{\text {eff }}^{2}$ is decreased down to a critical value, $\rho(0)$ vanishes - Fig. 3 - and for lower values of $\mu_{\text {eff }}^{2}$ the support of the distribution breaks down into two disconnected components (Fig. 4).

The arguments underlying the derivation in [64] are expected to be stronger in the fourdimensional case than in the two-dimensional setting, because in $D=2$ the dominance of the planar diagrams over the non-planar ones is weaker; however, even in $D=2$, one can still look for a signature of the expected effects - and, as it will be discussed in Section 3, the agreement with the numerical results appears quite remarkable.

A different model was studied in [54]: it is a scalar field theory in three-dimensional space, in which two coordinates are regularised using a fuzzy sphere, while the third one (labelled as $t$ ) is considered as purely commutative, and is regularised via a conventional lattice discretisation:

$$
\begin{aligned}
S(\Phi)= & \frac{4 \pi R^{2} \Delta t}{N} \sum_{t=1}^{N_{t}} \operatorname{tr}\left\{\frac{1}{2 R^{2}} \Phi(t)\left[L_{i},\left[L_{i}, \Phi(t)\right]\right]\right. \\
& \left.+\frac{1}{2}\left[\frac{\Phi(t+\Delta t)-\Phi(t)}{\Delta t}\right]^{2}+\frac{m^{2}}{2} \Phi^{2}(t)+\frac{\lambda}{4} \Phi^{4}(t)\right\} .
\end{aligned}
$$




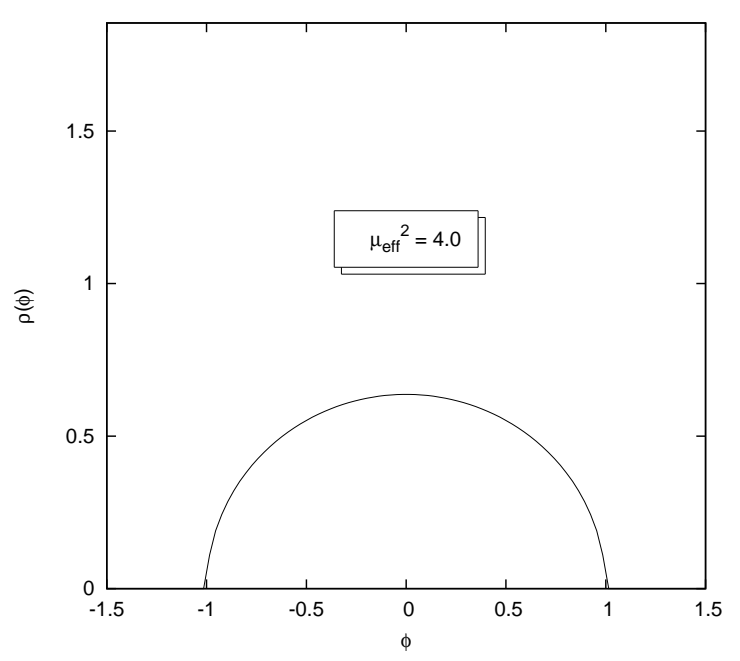

Figure 1. Eigenvalue distribution corresponding to Wigner's semi-circle law: this is obtained setting $\mu_{\text {eff }}^{2}=4$ in equation (6).

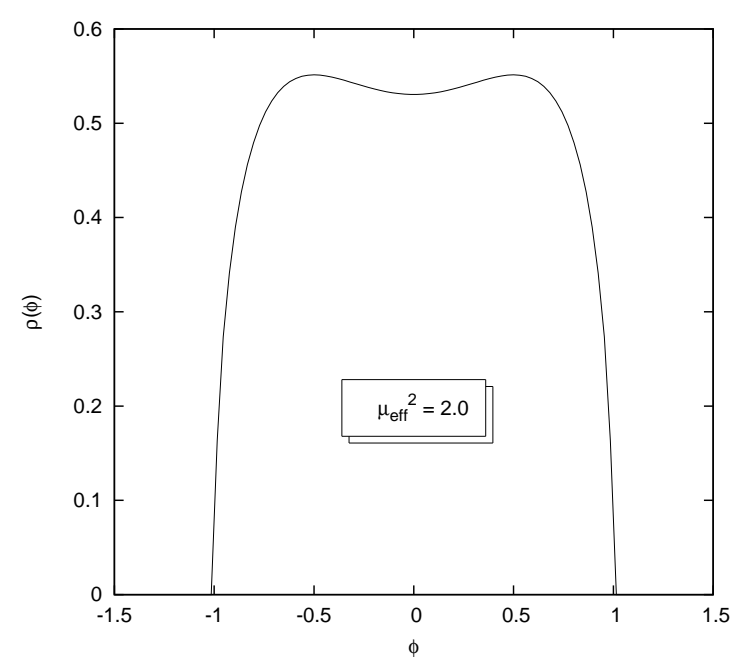

Figure 2. Typical profile of the eigenvalue distribution in the "one-cut phase".

Also in this case, different limits can be investigated, and one may expect a general pattern qualitatively similar to the lower-dimensional case discussed above.

On the other hand, a fuzzy space description for gauge theory was studied in some papers, including [65, 66, 67, 68, 69, 70, 71, 72, 73, 74, 75, 76, 58].

Supersymmetric versions of these models have been proposed, too. In particular, a supersymmetric generalisation of the fuzzy sphere was discussed in [77, 78, 79, 80], essentially based on the $\operatorname{osp}(2 \mid 1)$ algebra. Other works where the interplay between supersymmetry and the features of fuzzy models were discussed include [81, 82, 83, 56, 71, 84, 85, 86].

\section{Focus on the scalar model in two dimensions: a comparison with numerical results}

The models described in Section 2 can be quantised in the path integral approach, defining the expectation values of generic observables $\mathcal{O}=\mathcal{O}\left(c_{l, m}\right)$ as:

$$
\langle\mathcal{O}\rangle=\frac{\int \Pi_{l, m} d c_{l, m} \mathcal{O}\left(c_{l, m}\right) e^{-S}}{\int \Pi_{l, m} d c_{l, m} e^{-S}},
$$

where the $c_{l, m}$ are the components of the $\Phi$ matrix in a given basis (e.g.: in the polarisation tensor one), and can be interpreted as the dynamical degrees of freedom of the matrix model.

The right-hand side of equation (8) can be evaluated perturbatively, or estimated numerically from Monte Carlo simulations, in an approach similar to standard lattice field theory computations, namely: via averages over a sample of (independent) configurations with statistical weight proportional to $\exp [-S(\Phi)]$.

In this section, we summarise the present status of numerical results for the models discussed above, with a particular attention to the scalar theory on the two-dimensional fuzzy sphere. Our aim is to discuss the general physical interpretation of these results; therefore, we encourage the readers to refer to the papers mentioned, for precise details about the results, and for most of the technical aspects associated with the simulations and with the measurement of different observables.

The real scalar model with quartic interactions on the fuzzy sphere was studied numerically first in [52], where it was pointed out that the model exhibits three different phases: a disorder 


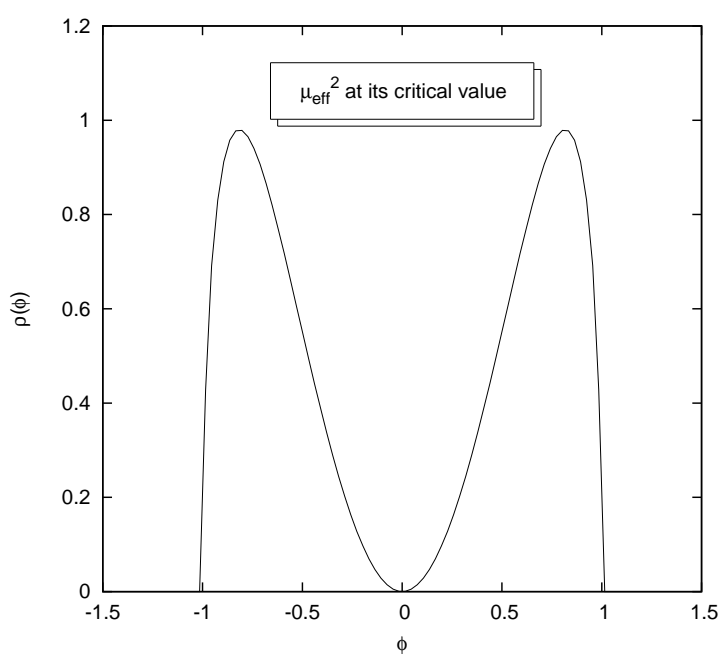

Figure 3. At the transition point, the probability to observe the zero eigenvalue becomes vanishing.

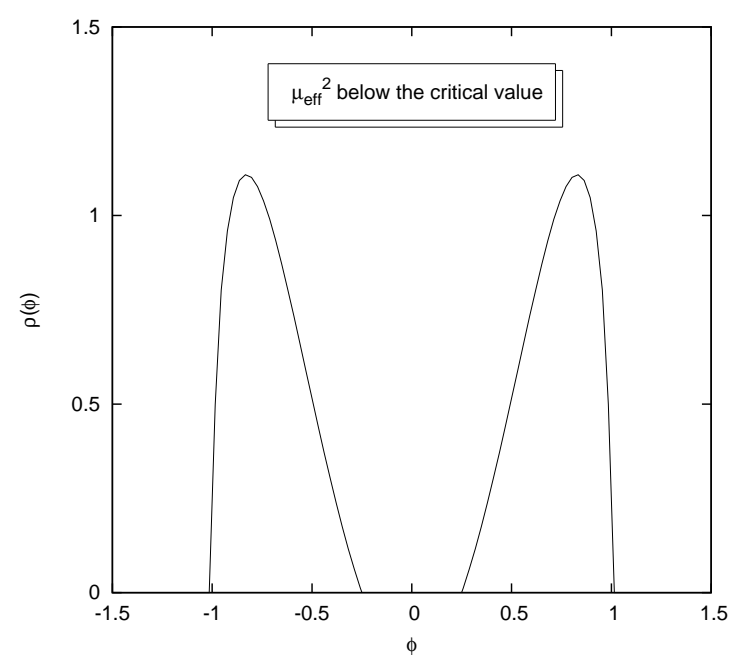

Figure 4. In the "two-cut phase", $\rho(\phi)$ is zero in a finite neighbourhood of the origin, and shows two disconnected peaks.

phase (with $\Phi$ fluctuating around zero), a uniform order phase (in which $\Phi$ typically fluctuates around either of the two classical minima of the potential), and the new, intermediate, nonuniform order phase. In particular, the critical line separating the disorder phase from the non-uniform order phase was identified, and the critical value for $\mu^{2}$ was found to scale like:

$$
\mu_{\mathrm{crit}}^{2} \simeq-\frac{0.56 N}{R} .
$$

In this work, it was also pointed out that the non-uniform order phase can be interpreted in terms of a pure potential model $[87,88]$, which can be obtained from equation (3) neglecting the kinetic term. The argument goes as follows: The minima of the potential term in equation (3) belong to orbits with representatives of the form:

$$
\Phi_{\min }^{(p)}=\sqrt{-\frac{6 \mu^{2}}{g}}\left(-\mathbb{1}_{p} \oplus \mathbb{1}_{N-p}\right), \quad \text { with: } \quad p \in\{0,1,2, \ldots, N\} .
$$

Unless the kinetic term lifts the degeneracy associated to the $p \in\{1,2, \ldots, N-1\}$ orbits, they are dominating - by virtue of their larger volume in the phase space - and imply non-maximal contributions to the expectation value of the matrix trace modulus ${ }^{2}$.

The results display a well-behaved collapse of data when $R^{2}$ is properly scaled, and are (qualitatively and approximately also quantitatively) consistent with the predictions in [64]: the non-perturbative data seem to lie in between the theoretical expectation of [64] (which, in $D=2$, is only an approximate one), and the pure potential model limit, in which the kinetic term is completely neglected.

The results of this study were later extended in [53], where large statistics were accumulated, in order to pin down the other critical line which appears in the phase diagram, namely: the one separating the non-uniform order from the uniform order phase. There, the phase diagram of the model was obtained, in terms of well-suited scaling combinations of the $\mu^{2}$ and $g$ parameters with powers of $N$. In particular, the two different transition lines meet at a triple point. In the large- $N$ limit, the critical line between the disorder and the non-uniform order regimes is expressed by a relation of the form: $g \propto\left(\mu_{\text {crit }}^{2}\right)^{2}$, whereas the transition curve separating the

\footnotetext{
${ }^{2}$ It is worth noting, however, that the dominating configurations in the model are actually those characterised by a smooth eigenvalue distribution.
} 


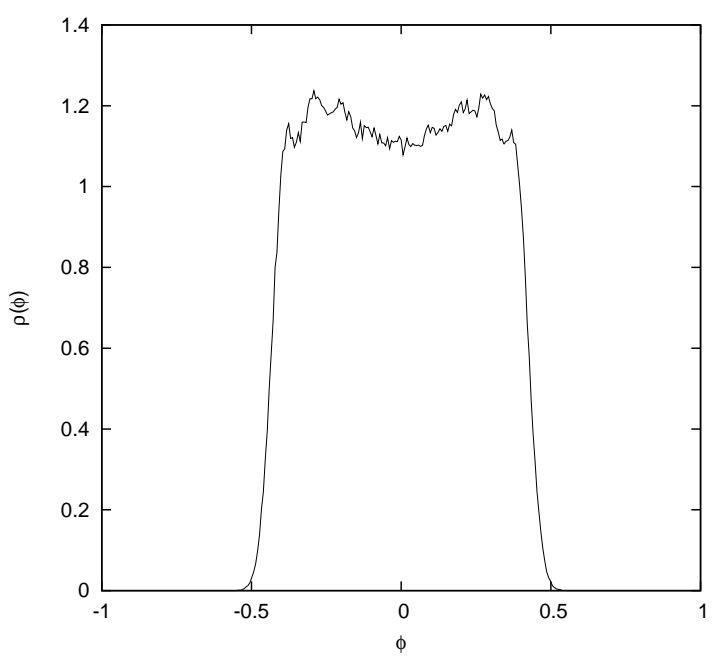

Figure 5. Numerical results for the eigenvalue distribution in the "one-cut" regime, from data obtained in preliminary, low-statistics runs with $N=9, R=1, g=972$ and $\mu^{2}=0$.

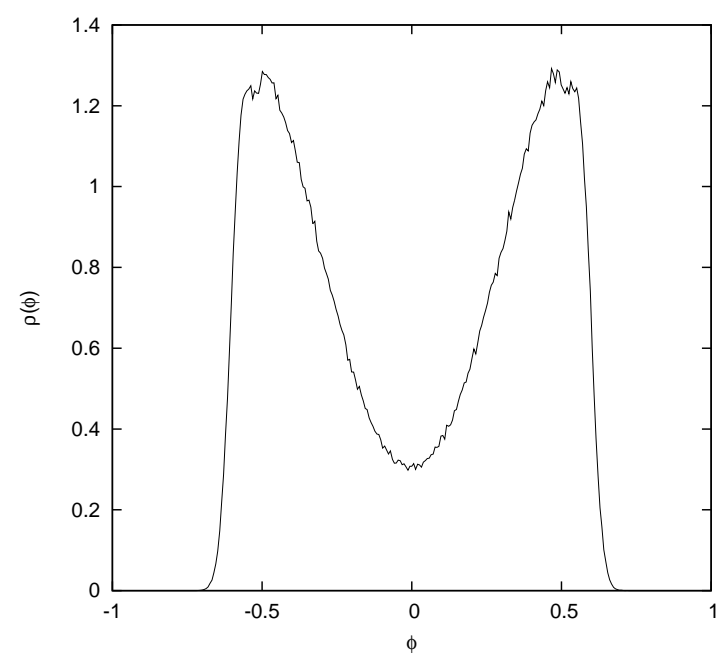

Figure 6. As $\mu^{2}$ is reduced and the transition point is approached, the eigenvalue density around $\phi=0$ decreases. The data shown correspond to $\mu^{2}=-74.25$, at the same $N, R$ and $g$ values as in Fig. 5.

uniform order from the non-uniform order regimes appears to be compatible with a straight line (remarkably, the collapse of data obtained in terms of the simple scaling laws of $g$ and $\mu^{2}$ works very well, even down to matrix sizes which are as small as $N=2$ ).

In the same paper, the behaviour of the specific heat per degree of freedom was worked out, and compared with the theoretical prediction from the large- $N$ limit of the pure potential model. The data for increasing matrix sizes appear to approach the expected behaviour, which would imply a discontinuity of the first kind in the derivative of the specific heat p.d.o.f. with respect to $\left(\frac{R \mu^{2}}{N \sqrt{g}}\right)$.

A recent work [59] has refined the numerical analysis of this model, by means of a new method, which reduces the correlation problems affecting simpler simulation algorithms. In the $\mu^{2}>0$ regime, this allowed to observe an indirect signature of the non-commutative anomaly, as a distortion of the dispersion relation for non-vanishing momenta. In the opposite regime, the high-precision numerical results allowed to highlight a more detailed interpretation of the matrix configurations associated with $l>0$ modes. In particular, the latter are not necessarily incompatible with a commutative setting: non-uniform configurations would also contribute the path integral of QFT on the finite-radius commutative sphere, and mediate the tunneling among the classical potential minima. Therefore, their signature in results of simulations in the large- $N$ limit at fixed $R$ is plausible, and not directly related to the UV/IR mixing.

The situation, of course, is different, once one investigates the double scaling limit; in fact, as we discussed above, the meaning of the latter consists in "removing" the regularisation cut-off $N$, while keeping the non-commutativity parameter fixed. In this case, the UV/IR mixing is expected (as the theory tends towards an approximation of the non-commutative plane), and should be responsible for effects which do not have a commutative counterpart. This description in the double scaling limit is also compatible with the picture of the pure potential model [87, 88], as it was discussed in $[52,53]$.

The best probe to detect the non-commutative effects in the regime where the transition from the disorder to the non-uniform order phase takes place is probably the density of the matrix eigenvalues. It is possible that different observables may be more appropriate for other regions in the parameter space, but at the moment a general theoretical understanding of the whole 


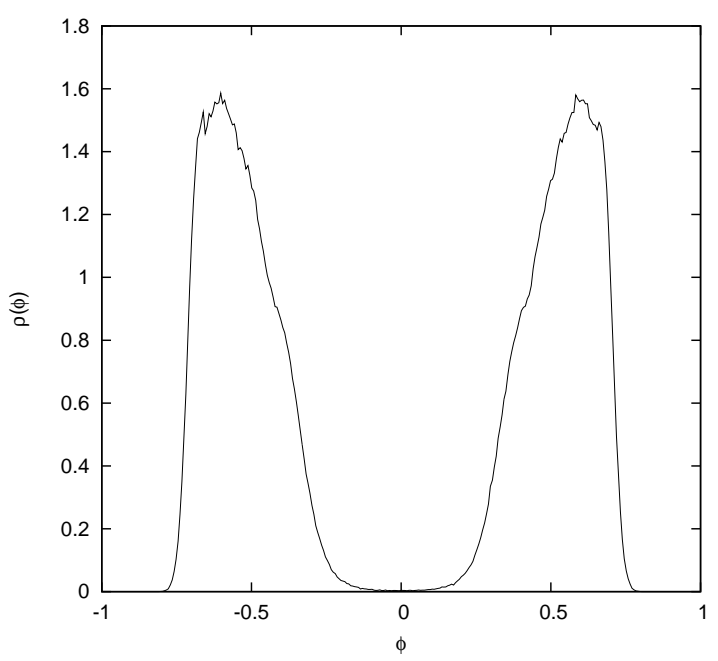

Figure 7. When $\mu^{2}$ reaches a critical value, $\rho(0)$ vanishes; the data shown here were obtained at $\mu^{2}=-114.75$.

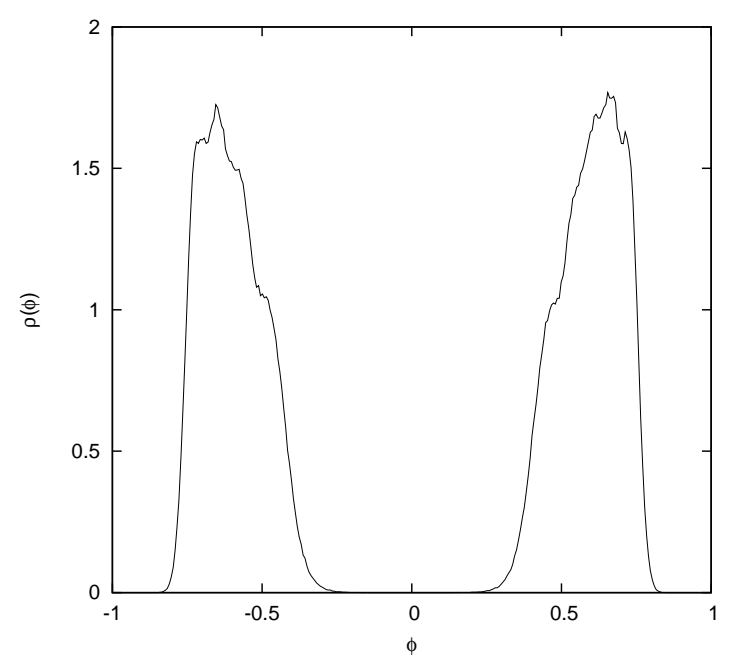

Figure 8. The simulation results below the transition point (the plot refers to data at $\mu^{2}=-135$ ) display a pattern compatible with the "two-cut phase" ...

phase diagram of the model is not consolidated yet. Here, we shall only consider the transition from the disorder to the non-uniform order phase: for this region, a clear theoretical prediction exists [64], which is formulated precisely in terms of the eigenvalue distribution. Technically, the measurement of the $\rho(\phi)$ distribution appears to be simpler than other observables which could allow to locate the phase transition, and an algorithm like the one used in [59], which is highly efficient in the exploration of the phase space of the model, allows to obtain significant results within a limited amount of CPU time.

In fact, the study of the eigenvalue distribution in the double-scaling limit is currently in progress, and the complete results will probably be published in an (upcoming) second version of that paper. The preliminary results obtained so far look very encouraging, showing a behaviour which confirms the predictions in [64].

As an example, in Figs. 5 to 10 we display the plots obtained from (low-statistics) independent data produced by test runs. These simulations correspond to $N=9$, holding the "non-commutativity parameter" $\frac{2 R^{2}}{N}=\frac{2}{9}$ fixed and for constant $g=972$, with $\mu^{2}$ taking four different, decreasing values. Note that the eigenvalues are not rescaled to the $[-1,1]$ range, therefore the normalisation for the $\phi$ axis is different with respect to [64] and to Figs. 1 to 4 . Also, the errorbars and complete details of the statistical analysis are not displayed for these preliminary data - they will be presented at the moment of the publication of the final results.

The eigenvalue distribution plotted in Fig. 5 is obtained for $\mu^{2}=0$ : its features appear to be compatible with the "one-cut" profile expected above the transition point (see Fig. 2).

For smaller values of $\mu^{2}$ (Fig. 6), the eigenvalue density around $\phi=0$ decreases, and appears to eventually vanish at a particular critical value: Fig. 7 shows the distribution profile approximately at the transition point ${ }^{3}$.

Below the critical value, a "two-cut" pattern emerges: the numerical results shown in Fig. 8 seem to confirm that $\rho(\phi)$ becomes peaked around two (symmetric) values, and, more important, that a gap of zero probability opens up for eigenvalues in a neighbourhood of zero. This is more

\footnotetext{
${ }^{3}$ It should be remarked that, in order to identify the transition properly, one should perform a study of the scaling properties, when the parameters are varied; this will be addressed elsewhere. Here, we shall not make any quantitative claims about the precise location of the critical lines. Still, the present discussion is (at least qualitatively) consistent with the expected theoretical pattern, and it provides the preliminary information necessary to address the complete study.
} 


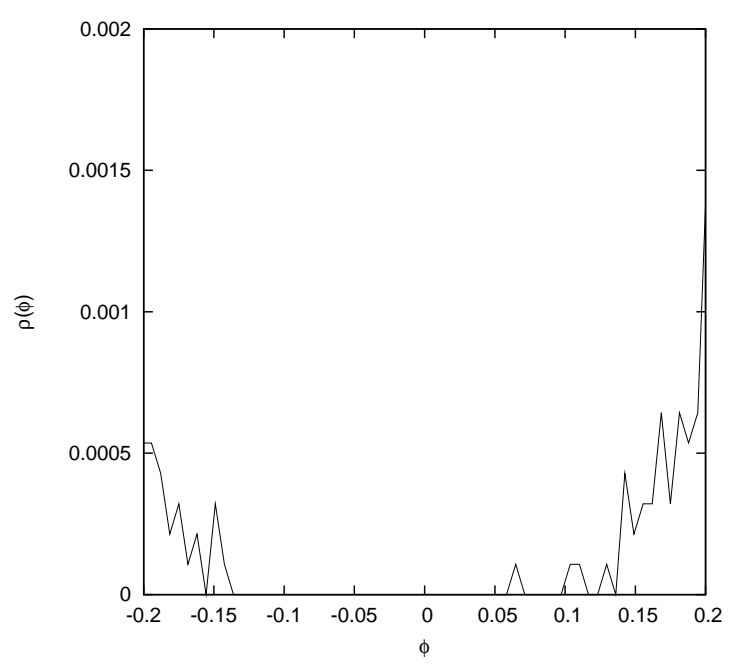

Figure 9. ... and a zero-density gap is observed in a finite interval around $\phi=0$.

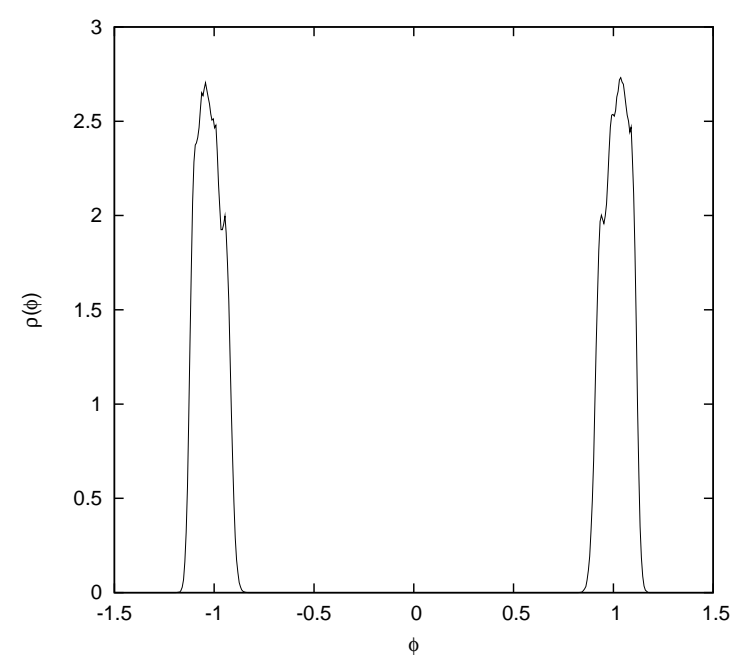

Figure 10. For $\mu^{2}$ values deeper in the "two-cut" regime $\left(\mu^{2}=-351\right)$, the distribution gets more localised around two narrow peaks, and a zoom to the origin of this figure gives stronger evidence of vanishing eigenvalue density in a (larger) neighbourhood of $\phi=0$ : a large range exists, in which no eigenvalues are observed.

clearly visible in Fig. 9, which is a zoom around the origin of the previous plot. Note, however, that in this case we are on the borderline of the statistical precision allowed by our sample, and the scale of the vertical axis is already comparable with the minimal resolution of the data.

In order to obtain more reliable information and see more precisely whether the eigenvalue distribution is indeed vanishing in this regime, it is instructive to extend the analysis to further, lower $\mu^{2}$ values. The result is displayed in Fig. 10, which confirms the pattern observed in Fig. 8, showing a distribution which is more strongly localised around two sharp peaks; a zoom to the origin shows - at least within the precision of the data - an exactly vanishing eigenvalue density in a larger neighbourhood of $\phi=0$.

Not surprisingly, the general qualitative features emerging from this picture are also shared by the three-dimensional model discussed in [54], in which two non-commutative directions are combined with a (compactified) commutative "time", and the regularised model is described by the action in equation (7). Indeed, also in that case, a phase was observed, which is unknown in the model in ordinary commutative space, and the different limits for $R$ and $N$ reproduce different physical theories.

\section{Conclusions}

To summarise the discussion presented above, we can conclude that the fuzzy space formulation encodes a very rich structure, describing different physical models.

On one hand, since the existence of "anomalous" effects, which have no commutative counterpart, shows that the naive formulation of the model would not reproduce the expected commutative theory, it would be interesting to address a detailed study of some "improved" version of the matrix model, which may yield the correct commutative limit.

On the other hand, the use of fuzzy spaces as a regularisation scheme for intrinsically noncommutative models appears to be one of the most powerful tools to investigate their nonperturbative features, both analytically and numerically. 
After reviewing the main problems in fuzzy models, we presented a preliminary sample of new numerical results, which are in agreement with the pattern predicted by the theory for the phase transition between two different regimes in the scalar model on the fuzzy sphere. In particular, we showed evidence supporting the picture of a phase transition which can be investigated looking at the topological properties of the matrix eigenvalue distribution.

Although in this paper we focused our attention onto some specific issues, the problems related to fuzzy spaces are among the main research interests of many different groups worldwide; therefore we apologise with the authors whose results have not been mentioned or properly discussed here. A lot of work has been done, and a lot more is currently in progress in this exciting research field - a field which, in view of the present status of the research, looks very promising.

\section{Acknowledgements}

The author thanks the organisers of the O'Raifeartaigh Symposium on Non-Perturbative and Symmetry Methods in Field Theory (June 22-24, 2006, Budapest, Hungary) for the stimulating and really enjoyable atmosphere at the conference, as well as A.P. Balachandran, W. Bietenholz, B.P. Dolan, K.S. Gupta, D. O'Connor and H. Steinacker for enlightening discussions. This work is supported by Enterprise Ireland under the Basic Research Programme.

[1] Doplicher S., Fredenhagen K., Roberts J.E., The quantum structure of space-time at the Planck scale and quantum fields, Comm. Math. Phys., 1995, V.172, 187-220, hep-th/0303037.

[2] Landi G., An introduction to noncommutative spaces and their geometry, hep-th/9701078.

[3] Douglas M.R., Nekrasov N.A., Noncommutative field theory, Rev. Modern Phys., 2001, V.73, 977-1029, hep-th/0106048.

[4] Szabo R.J., Symmetry, gravity and noncommutativity, hep-th/0606233.

[5] Karabali D., Nair V.P., Randjbar-Daemi S., Fuzzy spaces, the M(atrix) model and the quantum Hall effect, hep-th/0407007.

[6] Connes A., Douglas M.R., Schwarz A.S., Noncommutative geometry and matrix theory: Compactification on tori, JHEP, 1998, 9802, 003, 35 pages, hep-th/9711162.

[7] Douglas M.R., Hull C.M., D-branes and the noncommutative torus, JHEP, 1998, N 2, Paper 008, 5 pages, hep-th/9711165.

[8] Alekseev A.Y., Recknagel A., Schomerus V., Non-commutative world-volume geometries: Branes on SU(2) and fuzzy spheres, JHEP, 1999, N 9, Paper 023, 20 pages, hep-th/9908040.

[9] Seiberg N., Witten E., String theory and noncommutative geometry, JHEP, 1999, N 9, Paper 032, 93 pages, hep-th/9908142.

[10] Kar S., Non-commutativity, zero modes and D-brane geometry, Nuclear Phys. B, 2000, V.577, 171-182, hep-th/9911251.

[11] Kar S., D-branes, cyclic symmetry and noncommutative geometry, Modern Phys. Lett. A, 2003, V.18, 10531065, hep-th/0006073.

[12] Chamseddine A.H., Felder G., Fröhlich J., Gravity in noncommutative geometry, Comm. Math. Phys., 1993, V.155, 205-217, hep-th/9209044.

[13] Groenewold H.J., On the principles of elementary quantum mechanics, Physica, 1946, V.12, 405-460.

[14] Moyal J.E., Quantum mechanics as a statistical theory, Proc. Cambridge Phil. Soc., 1949, V.45, 99-124.

[15] Szabo R.J., Quantum field theory on noncommutative spaces, Phys. Rept., 2003, V.378, 207-299, hep-th/0109162.

[16] Doplicher S., Fredenhagen K., Roberts J.E., Space-time quantization induced by classical gravity, Phys. Lett. B, 1994, V.331, 39-44.

[17] Doplicher S., Spacetime and fields, a quantum texture, hep-th/0105251.

[18] Filk T., Divergencies in a field theory on quantum space, Phys. Lett. B, 1996, V.376, 53-58.

[19] Chen G.H., Wu Y.S., On critical phenomena in a noncommutative space, hep-th/0103020. 
[20] Jain A., Joglekar S.D., Causality violation in non-local quantum field theory, Internat. J. Modern Phys. A, 2004, V.19, 3409-3426, hep-th/0307208.

[21] Chaichian M., Kulish P.P., Nishijima K., Tureanu A., On a Lorentz-invariant interpretation of noncommutative space-time and its implications on noncommutative QFT, Phys. Lett. B, 2004, V.604, 98-102, hep-th/0408069.

[22] Minwalla S., Van Raamsdonk M., Seiberg N., Noncommutative perturbative dynamics, JHEP, 2000, N 2, Paper 020, 31 pages, hep-th/9912072.

[23] Hinchliffe I., Kersting N., Ma Y.L., Review of the phenomenology of noncommutative geometry, Internat. J. Modern Phys. A, 2004, V.19, 179-204, hep-ph/0205040.

[24] Gubser S.S., Sondhi S.L., Phase structure of non-commutative scalar field theories, Nuclear Phys. B, 2001, V.605, 395-424, hep-th/0006119.

[25] Castorina P., Zappalà D., Nonuniform symmetry breaking in noncommutative $\lambda \Phi^{4}$ theory, Phys. Rev. D, 2003, V.68, 065008, 7 pages, hep-th/0303030.

[26] Langmann E., Szabo R.J., Duality in scalar field theory on noncommutative phase spaces, Phys. Lett. B, 2002, V.533, 168-177, hep-th/0202039.

[27] Govindarajan T.R., Kürkçüoğlu S., Panero M., Nonlocal regularisation of noncommutative field theories, Modern Phys. Lett. A, 2006, V.21, 1851-1863, hep-th/0604061.

[28] Grosse H., Wulkenhaar R., Renormalisation of $\phi^{4}$-theory on noncommutative $\mathbb{R}^{4}$ in the matrix base, Comm. Math. Phys., 2005, V.256, 305-374, hep-th/0401128.

[29] Rivasseau V., Vignes-Tourneret F., Wulkenhaar R., Renormalization of noncommutative $\phi^{4}$-theory by multiscale analysis, Comm. Math. Phys., 2006, V.262, 565-594, hep-th/0501036.

[30] Grosse H., Steinacker H., Renormalization of the noncommutative $\phi^{3}$ model through the Kontsevich model, Nuclear Phys. B, 2006, V.746, 202-226, hep-th/0512203.

[31] Grosse H., Steinacker H., A nontrivial solvable noncommutative $\phi^{3}$ model in 4 dimensions, hep-th/0603052.

[32] Grosse H., Steinacker H., Exact renormalization of a noncommutative $\phi^{3}$ model in 6 dimensions, hep-th/0607235.

[33] Ambjørn J., Makeenko Y.M., Nishimura J., Szabo R.J., Finite $N$ matrix models of noncommutative gauge theory, JHEP, 1999, N 11, Paper 029, 17 pages, hep-th/9911041.

[34] Ambjørn J., Makeenko Y.M., Nishimura J., Szabo R.J., Nonperturbative dynamics of noncommutative gauge theory, Phys. Lett. B, 2000, V.480, 399-408, hep-th/0002158.

[35] Ambjørn J., Makeenko Y.M., Nishimura J., Szabo R.J., Lattice gauge fields and discrete noncommutative Yang-Mills theory, JHEP, 2000, N 5, Paper 023, 49 pages, hep-th/0004147.

[36] Azuma T., Bal S., Nagao K., Nishimura J., Nonperturbative studies of fuzzy spheres in a matrix model with the Chern-Simons term, JHEP, 2004, N 5, Paper 005, 36 pages, hep-th/0401038.

[37] Bietenholz W., Hofheinz F., Nishimura J., The renormalizability of 2D Yang-Mills theory on a noncommutative geometry, JHEP, 2002, N 9, Paper 009, 18 pages, hep-th/0203151.

[38] Bietenholz W., Hofheinz F., Nishimura J., Simulating non-commutative field theory, Nuclear Phys. Proc. Suppl., 2003, V.119, 941-946, hep-lat/0209021.

[39] Ambjørn J., Catterall S., Stripes from (noncommutative) stars, Phys. Lett. B, 2002, V.549, 253-259, hep-lat/0209106.

[40] Bietenholz W., Hofheinz F., Nishimura J., Phase diagram and dispersion relation of the non-commutative lambda $\phi^{4}$ model in $d=3$, JHEP, 2004, N 6, Paper 042, 36 pages, hep-th/0404020.

[41] Bietenholz W., Bigarini A., Hofheinz F., Nishimura J., Susaki Y., Volkholz J., Numerical results for U(1) gauge theory on 2d and 4d non-commutative spaces, Fortsch. Phys., 2005, V.53, 418-425, hep-th/0501147.

[42] Bietenholz W., Nishimura J., Susaki Y., Volkholz J., A non-perturbative study of 4d U(1) non-commutative gauge theory - the fate of one-loop instability, hep-th/0608072.

[43] Madore J., The fuzzy sphere, Classical Quantum Gravity, 1992, V.9, 69-87.

[44] Alexanian G., Balachandran A.P., Immirzi G., Ydri B., Fuzzy $\mathbb{C P}^{2}$, J. Geom. Phys., 2002, V.42, 28-53, hep-th/0103023.

[45] Balachandran A.P., Dolan B.P., Lee J.H., Martin X., O'Connor D., Fuzzy complex projective spaces and their star-products, J. Geom. Phys., 2002, V.43, 184-204, hep-th/0107099.

[46] Hammou A.B., Lagraa M., Sheikh-Jabbari M.M., Coherent state induced star-product on $\mathbb{R}_{\lambda}^{3}$ and the fuzzy sphere, Phys. Rev. D, 2002, V.66, 025025, 11 pages, hep-th/0110291. 
[47] Medina J., O'Connor D., Scalar field theory on fuzzy $S^{4}$, JHEP, 2003, N 11, Paper 051, 13 pages, hep-th/0212170.

[48] Vaidya S., Ydri B., On the origin of the UV-IR mixing in noncommutative matrix geometry, Nuclear Phys. B, 2003, V.671, 401-431, hep-th/0305201.

[49] Dolan B.P., O'Connor D., A fuzzy three sphere and fuzzy tori, JHEP, 2003, N 10, Paper 060, 16 pages, hep-th/0306231.

[50] Balachandran A.P., Kürkçüoğlu S., Vaidya S., Lectures on fuzzy and fuzzy SUSY physics, hep-th/0511114.

[51] Sheikh-Jabbari M.M., Inherent holography in fuzzy spaces and an $N$-tropic approach to the cosmological constant problem, hep-th/0605110.

[52] Martin X., A matrix phase for the $\phi^{4}$ scalar field on the fuzzy sphere, JHEP, 2004, N 4, Paper 077,20 pages, hep-th/0402230.

[53] García Floresn F., O'Connor D., Martin X., Simulating the scalar field on the fuzzy sphere, in Proceedings for the XXIIIrd International Symposium on Lattice Field Theory, PoS LAT2005, 2006, 262, 6 pages, hep-lat/0601012.

[54] Medina J., Bietenholz W., Hofheinz F., O'Connor D., Field theory simulations on a fuzzy sphere: an alternative to the lattice, in Proceedings for the XXIIIrd International Symposium on Lattice Field Theory, PoS LAT2005, 2006, 263, 6 pages, hep-lat/0509162.

[55] Azuma T., Bal S., Nagao K., Nishimura J., Absence of a fuzzy $S^{4}$ phase in the dimensionally reduced 5d Yang-Mills-Chern-Simons model, JHEP, 2004, N 7, Paper 066, 11 pages, hep-th/0405096.

[56] Anagnostopoulos K.N., Azuma T., Nagao K., Nishimura J., Impact of supersymmetry on the nonperturbative dynamics of fuzzy spheres, JHEP, 2005, N 9, Paper 046, 27 pages, hep-th/0506062.

[57] Azuma T., Bal S., Nagao K., Nishimura J., Perturbative versus nonperturbative dynamics of the fuzzy $S^{2} \times S^{2}$, JHEP, 2005, N 9, Paper 047, 20 pages, hep-th/0506205.

[58] O'Connor D., Ydri B., Monte Carlo simulation of a NC gauge theory on the fuzzy sphere, hep-lat/0606013.

[59] Panero M., Numerical simulations of a non-commutative theory: the scalar model on the fuzzy sphere, hep-th/0608202.

[60] Grosse H., Klimčík C., Prešnajder P., Towards finite quantum field theory in noncommutative geometry, Internat. J. Theor. Phys., 1996, V.35, 231-244, hep-th/9505175.

[61] Chu C.S., Madore J., Steinacker H., Scaling limits of the fuzzy sphere at one loop, JHEP, 2001, N 8, Paper 038, 17 pages, hep-th/0106205.

[62] Dolan B.P., O'Connor D., Prešnajder P., Matrix $\phi^{4}$ models on the fuzzy sphere and their continuum limits, JHEP, 2002, N 3, Paper 013, 15 pages, hep-th/0109084.

[63] Dolan B.P., O'Connor D., Private communication.

[64] Steinacker H., A non-perturbative approach to non-commutative scalar field theory, JHEP, 2005, N 3, Paper 075, 39 pages, hep-th/0501174.

[65] Klimčík C., Gauge theories on the noncommutative sphere, Comm. Math. Phys., 1998, V.199, 257-279, hep-th/9710153.

[66] Carow-Watamura U., Watamura S., Noncommutative geometry and gauge theory on fuzzy sphere, Comm. Math. Phys., 2000, V.212, 395-413, hep-th/9801195.

[67] Alekseev A.Y., Recknagel A., Schomerus V., Brane dynamics in background fluxes and non-commutative geometry, JHEP, 2000, N 5, Paper 010, 25 pages, hep-th/0003187.

[68] Hashimoto K., Krasnov K., D-brane solutions in non-commutative gauge theory on fuzzy sphere, Phys. Rev. D, 2001, V.64, 046007, 11 pages, hep-th/0101145.

[69] Kimura Y., Noncommutative gauge theories on fuzzy sphere and fuzzy torus from matrix model, Prog. Theoret. Phys., 2001, V.106, 445-469, hep-th/0103192.

[70] Steinacker H., Quantized gauge theory on the fuzzy sphere as random matrix model, Nuclear Phys. B, 2004, V.679, 66-98, hep-th/0307075.

[71] Iso S., Umetsu H., Note on gauge theory on fuzzy supersphere, Phys. Rev. D, 2004, V.69, 105014, 7 pages, hep-th/0312307.

[72] Kimura Y., Nonabelian gauge field and dual description of fuzzy sphere, JHEP, 2004, N 4, Paper 058,29 pages, hep-th/0402044.

[73] Azuma T., Bal S., Nagao K., Nishimura J., Dynamical aspects of the fuzzy $\mathbf{C P}^{2}$ in the large $N$ reduced model with a cubic term, JHEP, 2006, N 5, Paper 061, 27 pages, hep-th/0405277. 
[74] Grosse H., Steinacker H., Finite gauge theory on fuzzy $\mathbb{C P}^{2}$, Nuclear Phys. B, 2005, V.707, 145-198, hep-th/0407089.

[75] Castro-Villarreal P., Delgadillo-Blando R., Ydri B., A gauge-invariant UV-IR mixing and the corresponding phase transition for U(1) fields on the fuzzy sphere, Nuclear Phys. B, 2005, V.704, 111-153, hep-th/0405201.

[76] Behr W., Meyer F., Steinacker H., Gauge theory on fuzzy $S^{2} \times S^{2}$ and regularization on noncommutative $\mathbb{R}^{4}$, JHEP, 2005, N 7, Paper 040, 38 pages, hep-th/0503041.

[77] Grosse H., Klimčík C., Prešnajder P., Field theory on a supersymmetric lattice, Comm. Math. Phys., 1997, V.185, 155-175, hep-th/9507074.

[78] Grosse H. Reiter G., The fuzzy supersphere, J. Geom. Phys., 1998, V.28, 349-383, math-ph/9804013.

[79] Balachandran A.P., Kürkçüoğlu S., Rojas E., The star product on the fuzzy supersphere, JHEP, 2002, N 7, Paper 056, 22 pages, hep-th/0204170.

[80] Balachandran A.P., Pinzul A., Qureshi B., SUSY anomalies break $\mathcal{N}=2$ to $\mathcal{N}=1$ : the supersphere and the fuzzy supersphere, JHEP, 2005, N 12, Paper 002, 14 pages, hep-th/0506037.

[81] Iso S., Kimura Y., Tanaka K., Wakatsuki K., Noncommutative gauge theory on fuzzy sphere from matrix model, Nuclear Phys. B, 2001, V.604, 121-147, hep-th/0101102.

[82] Iso S., Umetsu H., Gauge theory on noncommutative supersphere from supermatrix model, Phys. Rev. D, 2004, V.69, 105003, 7 pages, hep-th/0311005.

[83] Hasebe K., Kimura Y., Fuzzy supersphere and supermonopole, Nuclear Phys. B, 2005, V.709, 94-114, hep-th/0409230.

[84] Kürkçüoğlu S., Non-linear sigma models on the fuzzy supersphere, JHEP, 2004, N 3, Paper 062 , 12 pages, hep-th/0311031.

[85] Imai T., Kitazawa Y., Takayama Y., Tomino D., Effective actions of matrix models on homogeneous spaces, Nuclear Phys. B, 2004, V.679, 143-167, hep-th/0307007.

[86] Imai T., Kitazawa Y., Takayama Y., Tomino D., Quantum corrections on fuzzy sphere, Nuclear Phys. B, 2003, V.665, 520-544, hep-th/0303120.

[87] Shimamune Y., On the phase structure of large N matrix models and gauge models, Phys. Lett. B, 1982, V.108, 407-410.

[88] Bleher P., Its A., Double scaling limit in the random matrix model: the Riemann-Hilbert approach, math-ph/0201003. 